\title{
Supporting Information for "A New Fluorescein Derivative Bearing Boronic Acid Group as a Fluorescent Chemosensor for Fluoride Ion"
}

K. M. K. Swamy, Yoon Ju Lee, Ha Na Lee, Jihyun Chun, Youngmee Kim, Sung-Jin Kim and Juyoung Yoon*

Department of Chemistry and Division of Nano Sciences, Ewha Womans University, 11-1

Daehyon-Dong, Sodaemun-Ku, Seoul 120-750, Korea

$$
\text { jyoon@ewha.ac.kr }
$$

$\mathrm{S}-$ Figure 1 S1 page

$\mathrm{S}-$ Figure 2 S2 page

$\mathrm{S}-$ Figure 3

S2 page

$\mathrm{S}-$ Figure 4

S3 page

$\mathrm{S}-$ Table 1

S4 page

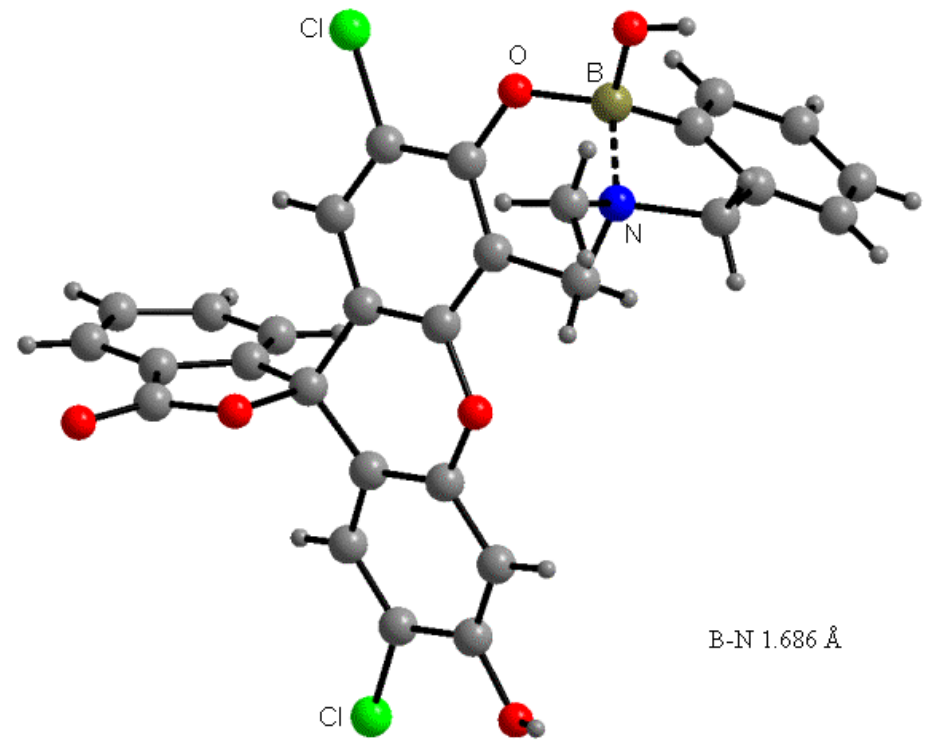

S-Figure 1. X-ray crystal structure of compound 1: Crystal Data for 1: $\mathrm{C}_{29} \mathrm{H}_{20} \mathrm{BCl}_{2} \mathrm{NO}_{8}, \mathrm{M}=592.17$, $\mathrm{P} 2_{1} / \mathrm{n}, \mathrm{a}=12.701(3) \AA, \mathrm{b}=14.224(3) \AA, \mathrm{c}=15.263(3) \AA, \beta=91.56(3)^{\circ}, \mathrm{V}=2756.2(9) \AA^{3}, \mathrm{Z}=4, \mu(\mathrm{Mo}$ $\mathrm{K} \alpha)=0.289 \mathrm{~mm}^{-1}, 13964$ reflections measured, 4834 unique $\left(\mathrm{R}_{\mathrm{int}}=0.1995\right)$ which were used in all calculations, final $R=0.1112\left(R_{w}=0.2902\right)$ with reflections having intensities greater than $2 \sigma, \operatorname{GOF}\left(F^{2}\right)$ $=0.965$. Structural information was deposited at the Cambridge Crystallographic Data Center (CCDC reference number 294725). 


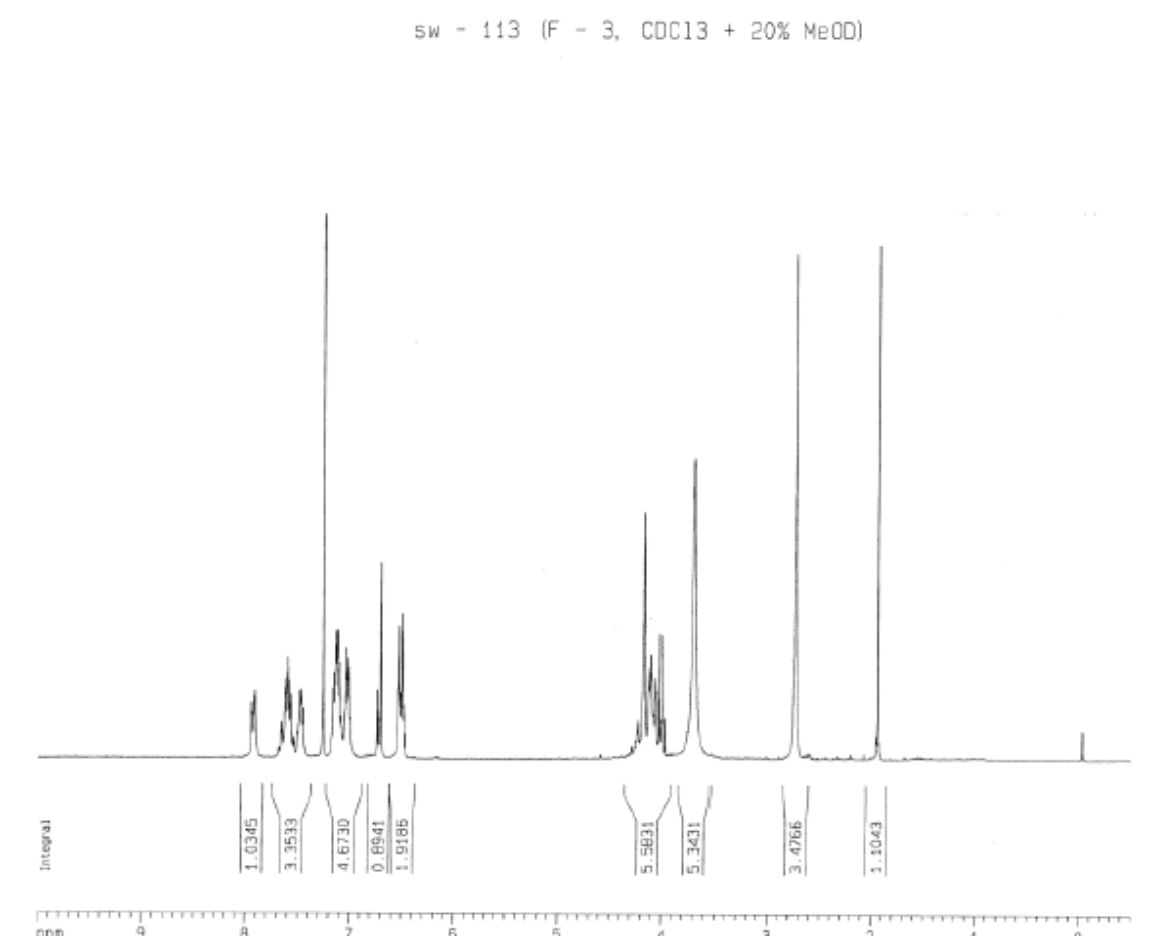

S-Figure 2. ${ }^{1} \mathrm{H} \mathrm{NMR}(250 \mathrm{MHz})$ spectrum of compound $\mathbf{1}$ in $\mathrm{CDCl}_{3}-\mathrm{CD}_{3} \mathrm{OD}(8: 2, \mathrm{v} / \mathrm{v})$.

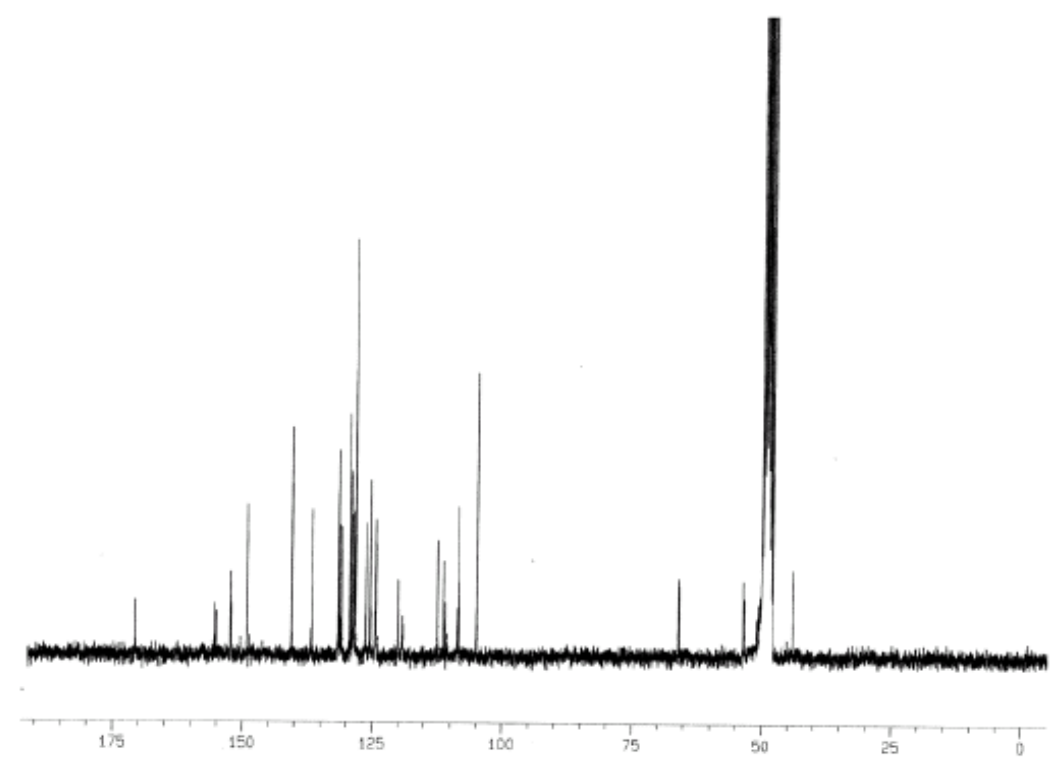

S-Figure 3. ${ }^{13} \mathrm{C}$ NMR (62.5 MHz) spectrum of compound $\mathbf{1}$ in $\mathrm{CD}_{3} \mathrm{OD}$. 
(b)

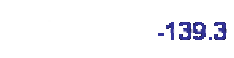

(a)

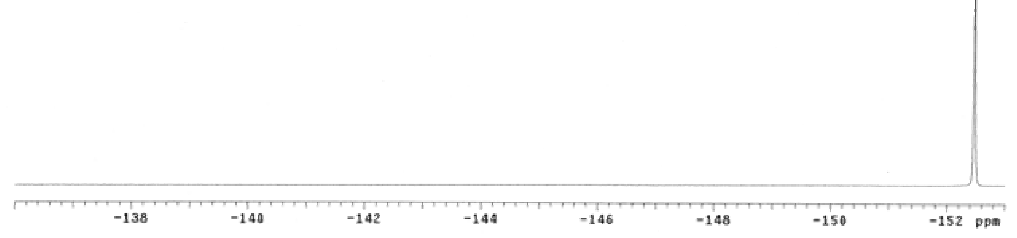

S-Figure 4. ${ }^{19} \mathrm{~F}$ NMR spectrum of compound $\mathbf{1}(2 \mathrm{mM})$ in $\mathrm{CD}_{3} \mathrm{OD}: \mathrm{CD}_{3} \mathrm{CN}(9: 1, \mathrm{v} / \mathrm{v})$, (a) $\mathbf{1}$ only; (b) $\mathbf{1}+$ $\mathrm{F}^{-}(1.1 \mathrm{eq})$. 
Table 1. Crystal data and structure refinement for $\mathbf{1}$.

Empirical formula

Formula weight

Temperature

Wavelength

Crystal system

Space group

Unit cell dimensions

Volume

$\mathrm{Z}$

Density (calculated)

Absorption coefficient

$\mathrm{F}(000)$

Crystal size

Theta range for data collection

Index ranges

Reflections collected

Independent reflections

Completeness to theta $=25.00$

Absorption correction

Refinement method

Data / restraints / parameters

Goodness-of-fit on F2

Final $\mathrm{R}$ indices $[\mathrm{I}>2 \operatorname{sigma}(\mathrm{I})]$

$\mathrm{R}$ indices (all data)

Largest diff. peak and hole

\section{C29 H20 B Cl2 N O8}

592.17

293(2) K

$0.71073 \AA$

Monoclinic

$\mathrm{P} 2(1) / \mathrm{n}$

$\mathrm{a}=12.701(3) \AA \quad \mathrm{a}=90.00^{\circ}$

$\mathrm{b}=14.224(3) \AA \quad \mathrm{b}=91.56(3)^{\circ}$

$\mathrm{c}=15.263(3) \AA \quad \mathrm{g}=90.00^{\circ}$

2756.2(9) $\AA^{3}$

4

$1.427 \mathrm{Mg} / \mathrm{m}^{3}$

$0.289 \mathrm{~mm}^{-1}$

1216

$0.25 \times 0.20 \times 0.20 \mathrm{~mm}^{3}$

1.96 to $25.00^{\circ}$.

$-7 \leq \mathrm{h} \leq 15,-16 \leq \mathrm{k} \leq 16,-18 \leq 1 \leq 16$

13964

$4834[\mathrm{R}(\mathrm{int})=0.1995]$

$99.8 \%$

None

Full-matrix least-squares on $\mathrm{F}^{2}$

4834 / 1 / 373

0.965

$\mathrm{R} 1=0.1112, \mathrm{wR} 2=0.2902$

$\mathrm{R} 1=0.2762, \mathrm{wR} 2=0.3784$

1.304 and -0.464 e. $\AA^{-3}$ 
Table 2. Selected bond lengths $[\AA]$ and angles $\left[{ }^{\circ}\right]$ for $\mathbf{1}$.

\begin{tabular}{|c|c|}
\hline $\mathrm{B}(1)-\mathrm{O}(6)$ & $1.427(14)$ \\
\hline $\mathrm{B}(1)-\mathrm{O}(2)$ & $1.472(13)$ \\
\hline $\mathrm{B}(1)-\mathrm{C}(21)$ & $1.579(16)$ \\
\hline $\mathrm{B}(1)-\mathrm{N}(1)$ & $1.686(15)$ \\
\hline $\mathrm{C}(1)-\mathrm{O}(1)$ & $1.380(11)$ \\
\hline $\mathrm{C}(3)-\mathrm{O}(2)$ & $1.322(11)$ \\
\hline $\mathrm{C}(4)-\mathrm{Cl}(1)$ & $1.733(10)$ \\
\hline $\mathrm{C}(7)-\mathrm{O}(4)$ & $1.516(12)$ \\
\hline $\mathrm{C}(10)-\mathrm{Cl}(2)$ & $1.735(11)$ \\
\hline $\mathrm{C}(13)-\mathrm{O}(1)$ & $1.371(12)$ \\
\hline $\mathrm{C}(14)-\mathrm{N}(1)$ & $1.510(12)$ \\
\hline $\mathrm{C}(15)-\mathrm{N}(1)$ & $1.512(13)$ \\
\hline $\mathrm{C}(22)-\mathrm{N}(1)$ & $1.461(13)$ \\
\hline $\mathrm{C}(29)-\mathrm{O}(4)$ & $1.311(11)$ \\
\hline $\mathrm{C}(29)-\mathrm{O}(5)$ & $1.317(2)$ \\
\hline $\mathrm{O}(6)-\mathrm{B}(1)-\mathrm{O}(2)$ & $106.4(9)$ \\
\hline $\mathrm{O}(6)-\mathrm{B}(1)-\mathrm{C}(21)$ & $114.7(9)$ \\
\hline $\mathrm{O}(2)-\mathrm{B}(1)-\mathrm{C}(21)$ & $122.7(9)$ \\
\hline $\mathrm{O}(6)-\mathrm{B}(1)-\mathrm{N}(1)$ & 108.1(9) \\
\hline $\mathrm{O}(2)-\mathrm{B}(1)-\mathrm{N}(1)$ & $107.5(8)$ \\
\hline $\mathrm{C}(21)-\mathrm{B}(1)-\mathrm{N}(1)$ & $96.0(8)$ \\
\hline $\mathrm{O}(1)-\mathrm{C}(1)-\mathrm{C}(6)$ & $123.2(9)$ \\
\hline $\mathrm{O}(1)-\mathrm{C}(1)-\mathrm{C}(2)$ & $114.7(9)$ \\
\hline $\mathrm{O}(2)-\mathrm{C}(3)-\mathrm{C}(2)$ & $122.3(9)$ \\
\hline $\mathrm{O}(2)-\mathrm{C}(3)-\mathrm{C}(4)$ & $120.6(9)$ \\
\hline $\mathrm{C}(8)-\mathrm{C}(7)-\mathrm{O}(4)$ & $105.8(8)$ \\
\hline $\mathrm{C}(6)-\mathrm{C}(7)-\mathrm{O}(4)$ & $106.9(8)$ \\
\hline $\mathrm{C}(23)-\mathrm{C}(7)-\mathrm{O}(4)$ & $102.3(8)$ \\
\hline $\mathrm{O}(3)-\mathrm{C}(11)-\mathrm{C}(12)$ & $123.0(10)$ \\
\hline $\mathrm{O}(3)-\mathrm{C}(11)-\mathrm{C}(10)$ & $118.0(10)$ \\
\hline $\mathrm{O}(1)-\mathrm{C}(13)-\mathrm{C}(8)$ & $121.6(10)$ \\
\hline $\mathrm{O}(1)-\mathrm{C}(13)-\mathrm{C}(12)$ & $115.7(9)$ \\
\hline $\mathrm{N}(1)-\mathrm{C}(14)-\mathrm{C}(2)$ & $111.9(8)$ \\
\hline
\end{tabular}




$$
\begin{array}{ll}
\mathrm{C}(16)-\mathrm{C}(15)-\mathrm{N}(1) & 103.9(8) \\
\mathrm{C}(16)-\mathrm{C}(21)-\mathrm{B}(1) & 109.0(9) \\
\mathrm{C}(20)-\mathrm{C}(21)-\mathrm{B}(1) & 132.6(10) \\
\mathrm{O}(4)-\mathrm{C}(29)-\mathrm{O}(5) & 118.8(9) \\
\mathrm{O}(4)-\mathrm{C}(29)-\mathrm{C}(28) & 113.3(8) \\
\mathrm{O}(5)-\mathrm{C}(29)-\mathrm{C}(28) & 127.9(10) \\
\mathrm{C}(22)-\mathrm{N}(1)-\mathrm{C}(14) & 110.3(8) \\
\mathrm{C}(22)-\mathrm{N}(1)-\mathrm{C}(15) & 113.9(9) \\
\mathrm{C}(14)-\mathrm{N}(1)-\mathrm{C}(15) & 106.2(8) \\
\mathrm{C}(22)-\mathrm{N}(1)-\mathrm{B}(1) & 115.8(8) \\
\mathrm{C}(14)-\mathrm{N}(1)-\mathrm{B}(1) & 109.0(7) \\
\mathrm{C}(15)-\mathrm{N}(1)-\mathrm{B}(1) & 100.9(8) \\
\mathrm{C}(13)-\mathrm{O}(1)-\mathrm{C}(1) & 117.9(8) \\
\mathrm{C}(3)-\mathrm{O}(2)-\mathrm{B}(1) & 121.5(8) \\
\mathrm{C}(29)-\mathrm{O}(4)-\mathrm{C}(7) & 108.2(7)
\end{array}
$$

